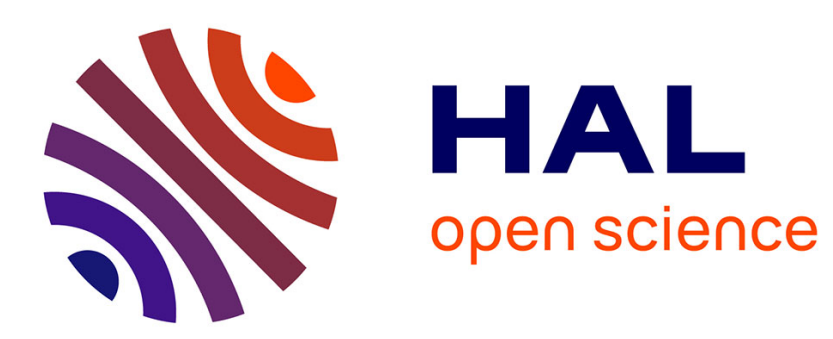

\title{
Le rôle de la famille dans la transmission politique entre les générations. Histoire et bilan des études de socialisation politique
}

Vincent Tournier

\section{- To cite this version:}

Vincent Tournier. Le rôle de la famille dans la transmission politique entre les générations. Histoire et bilan des études de socialisation politique. Politiques sociales et familiales, 2009, 99, pp.59-72. halshs-00578040

\author{
HAL Id: halshs-00578040 \\ https://shs.hal.science/halshs-00578040
}

Submitted on 18 Mar 2011

HAL is a multi-disciplinary open access archive for the deposit and dissemination of scientific research documents, whether they are published or not. The documents may come from teaching and research institutions in France or abroad, or from public or private research centers.
L'archive ouverte pluridisciplinaire HAL, est destinée au dépôt et à la diffusion de documents scientifiques de niveau recherche, publiés ou non, émanant des établissements d'enseignement et de recherche français ou étrangers, des laboratoires publics ou privés. 


\title{
Le rôle de la famille dans la transmission politique entre les générations
} Histoire et bilan des études de socialisation politique

\author{
\begin{tabular}{l|l} 
& \\
Vincent Tournier & Maître de conférences en sciences politiques
\end{tabular} \\ (IEP de Grenoble - PACTE).
}

Mots-clés : Socialisation politique - Rôle politique de la famille - Transmission familiale.

\begin{abstract}
Cet article propose de dresser un bilan des études consacrées à l'influence politique de la famille. Après un retour sur la genèse des débats contemporains, il montre que l'avènement d'une réflexion de nature scientifique s'est fait de manière progressive après la Seconde Guerre mondiale dans le cadre des recherches sur la socialisation politique des enfants. Dans une première phase, que l'on pourrait qualifier de "préscientifique ", les approches dominantes ont été relativement catégoriques. Les discussions qui ont suivi ont, cependant, conduit à remettre en cause les certitudes initiales, notamment sur la place de l'enfance et l'impact réel de la famille dans la transmission des opinions politiques. Ce faisant, la réflexion s'en est trouvée enrichie, ce qui permet aujourd'hui d'envisager de nouvelles pistes de recherche.
\end{abstract}

I est difficile de trouver une idée plus répandue que celle qui attribue à la famille un rôle décisif dans la transmission des opinions politiques entre les générations. Cette idée n'est pas nouvelle, mais sait-on précisément d'où elle vient et quelles en sont les bases théoriques? Aujourd'hui, après un demi-siècle de recherches, où en est-on ?

La réflexion sur le rôle politique de la famille est indissociable d'un champ de recherche apparu aux États-Unis dans les années 1950-1960, qui a suscité beaucoup d'espoirs avant de rencontrer d'importantes critiques : la socialisation politique. À l'origine, l'objectif était de reprendre une question aussi vieille que la philosophie - celle de l'éducation et de la formation des citoyens - en adoptant un point de vue qui se voulait strictement descriptif, en rupture avec les approches normatives ou moralistes du passé (Percheron, 1974). Il s'agissait non plus de réfléchir sur la meilleure façon de faire de "bons» citoyens, mais d'étudier les processus concrets par lesquels se forment et se transforment les opinions politiques individuelles. Les conclusions devaient reposer non pas sur des intuitions ou des jugements de valeur, mais sur des observations vérifiables et cumulables. Dès le début, l'attention des chercheurs s'est portée sur la famille, qui a très vite été considérée comme le principal agent (agency) de socialisation politique. Ce n'était toutefois pas la première fois que le rôle politique de la famille se voyait ainsi proclamé. La thèse du rôle politique de la famille est un grand classique de la pensée politique car la famille a été fortement mobilisée par les idéologies politiques. Cette " politisation » de la famille tient au fait que, à travers la famille, un débat majeur s'est joué, qui n'est autre que le débat fondateur de la modernité puisqu'il concerne la définition même de l'autorité politique et renvoie, de ce fait, à la question fondamentale de la nature humaine.

C'est probablement pour avoir oublié cet arrièrefond idéologique que l'entrée de la socialisation politique dans la maturité scientifique s'est révélée plus délicate que prévu. Les premières recherches n'ont pas toujours été très rigoureuses dans leur méthode comme dans leur questionnement. Durant cette phase initiale - que l'on pourrait qualifier de "préscientifique »-, l'aspiration à l'objectivité a été contrariée par la prégnance de présupposés moralistes hérités des préoccupations antérieures. Cette phase de tâtonnements s'est traduite par une succession de paradigmes [au sens de Thomas Kuhn, 1983(1962)], dont la validité scientifique n'a guère été démontrée. Du coup, les critiques ont rapidement balayé les belles assurances du début. Cette phase de déconstruction a fortement mis à mal les recherches sur la socialisation politique, mais elle a également permis de renouveler la réflexion. Ce sont ces différentes étapes que l'on retrace dans cet article, de manière à mieux cerner les enjeux des recherches à venir.

Cet article est une version remaniée du chapitre paru dans l'ouvrage collectif : Quéniart A. et Hurtubise R (dir), 2009, L'intergénérationnels Regards pluridisciplinaires, Rennes, Presses de I'EHESP, lien social et Politiques. 


\section{La famille comme enjeu idéologique}

D'où vient l'intérêt pour le rôle politique de la famille? Pour comprendre les origines de ce débat, il faut remonter assez loin dans I'histoire, lorsque la famille s'est trouvée au cœur des grands affrontements sur la définition de l'autorité politique.

\section{La définition de l'autorité politique}

Les théoriciens de I'absolutisme royal ont souvent été tentés de prendre la famille comme point de départ pour justifier l'autorité du roi. En Angleterre, dans Patriarcha ou du pouvoir naturel des rois [1991 (1640)], sir Robert Filmer s'est appuyé sur les Écritures pour condamner l'idée que les peuples peuvent déposer leurs Princes. Si une telle éventualité est à ses yeux inconcevable, c'est parce que le pouvoir du roi dérive de l'autorité paternelle, qui a ellemême été originellement léguée par Dieu à Adam. Pour R. Filmer, la condition naturelle des hommes est d'être assujettis à leurs pères, puis à leur roi. Ces idées ont été reprises en France par JacquesBénigne Bossuet qui, dans la Politique tirée des propres paroles de l'Écriture sainte [1864(1709)], soutient à son tour que "le premier empire parmi les hommes est l'empire paternel", de sorte que "la première idée de commandement et d'autorité humaine est venue aux hommes de l'autorité paternelle» (Bossuet, 1864:96).

En plaçant la famille au cœur de leur argumentation, les théoriciens de l'absolutisme ont cru bâtir une défense inattaquable. Ils se sont trompés, car $\mathrm{C}^{\prime}$ est précisément sur elle que vont se concentrer les critiques des théoriciens du contrat social (Borgetto, 1983). La base de I'argumentation est posée par John Locke avant d'être reprise par Jean-Jacques Rousseau. Dans son Traité du gouvernement civil [1992(1690)], J. Locke affirme sa volonté d'anéantir la théorie de R. Filmer, comme l'affiche explicitement le sous-titre de son livre ("The false principles and foundations of Sir Robert Filmer and is followers are detected and overthrown »). Sa démarche consiste à déconstruire l'autorité absolue du père pour mieux saper les fondements de l'absolutisme. Tout d'abord, J. Locke fait remarquer que l'autorité dans la famille est nécessairement partagée entre le père et la mère car l'enfant appartient à ses deux parents (le Décalogue ne dit-il pas «honore ton père et ta mère » ?). Il précise ensuite que l'autorité paternelle ne saurait être sans borne: non seulement cette autorité est limitée dans le temps (elle ne vaut que jusqu'à " l'âge de raison » des enfants) mais, de plus, même durant la minorité des enfants, le pouvoir du père ne saurait être absolu car une chose est de demander aux enfants de respecter et $\mathrm{d}^{\prime}$ honorer ses parents, autre chose est de les obliger "à une obéissance et à une soumission absolues". Rien ne permet donc de prétendre que l'autorité appartient, par nature, à une seule personne. Or, ce qui est vrai pour la famille l'est aussi pour la société dans son ensemble. Le roi ne saurait gouverner en vertu d'une loi qui préexiste à la société et sur laquelle les hommes n'auraient pas leur mot à dire. Les lois ne sont pas fixées par avance; elles sont déterminées par des hommes libres et égaux car toute société est le fruit d'une union volontaire. $C^{\prime}$ est donc aux hommes eux-mêmes qu'il revient de déterminer, sous la forme d'un contrat, la forme de l'autorité et l'organisation du pouvoir.

\section{Familialisme versus individualisme}

Ce débat, qui n'est autre que le débat fondateur de la démocratie, a jeté les bases d'un clivage qui va perdurer jusqu'à nos jours. En forçant un peu le trait, on peut dire qu'il oppose deux conceptions. La première voit dans la famille une réalité naturelle, fondée sur des lois qui préexistent aux individus. La seconde défend, au contraire, la primauté de l'individu sur la famille, donc la priorité des droits sur les devoirs, elle insiste sur l'autonomie de la personne, qui constitue la seule valeur morale authentique. En France, cet affrontement a pris une ampleur particulière pendant la Révolution de 1789. L'affirmation des droits de l'homme, l'élaboration d'une nouvelle législation civile marquant à la fois la fin de la puissance paternelle, I'instauration du divorce et l'égalité entre les enfants, ont déchaîné les passions. Les auteurs contre-révolutionnaires, Louis de Bonald à leur tête, récusent les principes de l'individualisme et n'ont eu de cesse de réaffirmer la primauté naturelle de la famille. À l'opposé des idées de J. Locke, pour qui l'individu est en mesure de s'émanciper de ses parents, les contre-révolutionnaires soutiennent que la famille joue un rôle déterminant dans la formation des enfants. Une thèse va ainsi connaître un important succès et préfigurer assez bien les réflexions à venir sur la socialisation politique : par l'intermédiaire de l'autorité paternelle, la famille est un lieu "d'accoutumance » à l'autorité. Les prémices de cette thèse avaient déjà été formulées par J.-B. Bossuet. "Les hommes naissent tous sujets, écrivait-il, et l'empire paternel, qui les accoutume à obéir, les accoutume en même temps à n'avoir qu'un chef » [Bossuet, 1864(1709)]. Si les hommes ressentent le besoin de faire des rois, c'est pour retrouver l'autorité paternelle qu'ils ont connue dans leur enfance. Cette idée est reprise au XIX' ${ }^{\mathrm{e}}$ siècle par des auteurs tels qu'Auguste Comte ou Frédéric Le Play, qui élaborent une quasi-théorie de la socialisation par la famille. A. Comte voit ainsi dans la famille une école de l'obéissance, une " école éternelle de la vie sociale » [Comte, 1975(1842)], tandis que F. Le Play conçoit la famille comme une «école de la paix sociale » ou une "école de la vie privée » voire, plus simplement, une « école sociale » [Le Play 1989(1879)]. Ceci explique I'importance que ces auteurs attachent à la préservation des familles : une 
société stable et prospère commence par une famille saine, placée sous l'affectueuse mais ferme autorité du père. Dans son éloge du royaume de Sardaigne, Joseph de Maistre avait déjà annoncé la couleur: la paix civile ne peut être garantie que lorsque " les fils de famille vivent paisiblement sous le joug salutaire qui les assujettit » [Maistre (de) 1989(1793-1798)].

Ces différents propos ont le mérite de mettre en lumière l'arrière-fond idéologique du débat. Ils montrent que si la famille fait l'objet d'investissements idéologiques intenses, c'est parce que les discours sur la famille s'inscrivent dans une vision plus générale de l'homme et de la société. Dans une étude consacrée aux idées sur la famille, Pierre Bréchon le résume très bien : "les idées familiales ne sont jamais des idées "en soi". Elles s'intègrent toujours dans un système de pensée; elles sont toujours dépendantes d'une conception de la société » (Bréchon, 1976:14). Et si la famille a pu servir de point d'appui à des préjugés idéologiques, c'est parce qu'un débat important se joue en arrièreplan: la question de l'autonomie de l'individu, c'est-à-dire sa capacité de se constituer en tant qu'être de raison et de conscience, indépendamment de toute détermination liée à son entourage ou à son environnement. Tel est, au fond, le principal dilemme auquel vont être confrontées les réflexions ultérieures sur la socialisation politique : comment penser l'influence politique de la famille sans tomber dans la négation de l'individu? Est-il possible de dépasser une vision pessimiste fondée sur la surdétermination par la famille, sans pour autant céder à la vision optimiste d'un individu purement rationnel, vierge de toute influence extérieure?

\section{Les premières théories : I'influence de la psychanalyse}

Entre les deux guerres mondiales, la situation politique de l'Europe a provoqué un regain d'intérêt pour le rôle politique de la famille. À travers la volonté de comprendre les ressorts du fascisme, les chercheurs de l'Institut de recherches sociales - créé à Francfort en 1929 puis transféré aux États-Unis ont engagé une réflexion qui a grandement inspiré les politistes de l'après-guerre.

\section{L'école de Francfort}

Si les traditionalistes ont vu dans la famille une école de la vie sociale, les philosophes de Francfort vont en faire une école de l'autorité. Dans les deux cas, la problématique demeure similaire: $c^{\prime}$ est dans la famille que s'effectuent les apprentissages politiques fondamentaux de l'individu, que se jouent, dès les premières années de l'existence, les relations ultérieures avec l'autorité. Telle est la thèse que I'on va retrouver dans un ouvrage aujourd'hui oublié, les Studien über Autorität und Familie, publié à Paris en 1936 sous la direction de Max Horkheimer, ouvrage qui préfigure le célèbre travail dirigé par Theodor Adorno The Authoritariam Personnality (Adorno et al., 1950).

L'originalité des théoriciens de l'école de Francfort est de fusionner Marx et Freud dans une même théorie, la "théorie critique ». Cette théorie se démarque du marxisme orthodoxe en ce sens qu'elle entend réviser à la baisse le rôle des facteurs économiques au profit des facteurs culturels tout en s'inspirant fortement de la psychanalyse pour formuler autrement la question de la domination. Leur idée est que, dans les sociétés capitalistes modernes, I'assujettissement des classes populaires est d'abord de nature psychologique car elle correspond à un " état de dépendance accepté ». Un travail $d^{\prime}$ inculcation est assuré par des institutions sociales (ou « médiatisantes») que sont l'école, l'église et, surtout, la famille, qui sont elles-mêmes façonnées par la structure économique mais qui ont acquis leur autonomie. Conçue comme un médiateur entre l'infrastructure matérielle et la superstructure idéologique, la famille a pour fonction de briser la volonté de l'enfant en lui inculquant "l'obligation intériorisée d'obéir inconditionnellement au devoir » (Horkheimer, 1974). Le système capitaliste génère donc la famille autoritaire. II «transforme le père en maître ", puisque le père est celui qui ramène I'argent et décide de son utilisation; sa femme et ses enfants doivent se soumettre à son commandement. Du coup, "le monde mental dans lequel l'enfant grandit [est dominé] par l'idée que certains hommes exercent un pouvoir sur d'autres, qu'il y a ce qui est en haut et ce qui est en bas, les ordres et l'obéissance » (Horkheimer, 1974:283-284). Ce déterminisme familial est d'autant plus fort que, comme l'enseigne la psychologie des profondeurs, tout se joue durant l'enfance. À partir de ce cadre théorique, M. Horkheimer présente le fascisme comme le produit d'une double crise : crise économique tout d'abord, avec notamment la montée du chômage, crise de la famille ensuite, qui résulte de l'effritement du prestige du père. À cause de cette crise, les enfants qui ont été socialisés dans un cadre familial autoritaire ont besoin d'une autorité de substitution, qui va être assurée par l'État ou par un chef charismatique. Cette interprétation du fascisme a été critiquée par les historiens en raison de son réductionnisme psychologique. II est néanmoins intéressant de remarquer à quel point la problématique des Studien fait écho à la pensée traditionaliste du XIXe siècle. La philosophie des Lumières fait d'ailleurs l'objet d'une critique systématique de la part de M. Horkheimer. Sont rejetés des penseurs tels que René Descartes, J. Locke et Emmanuel Kant, promoteurs $\mathrm{d}^{\prime}$ une « pensée bourgeoise » qui consiste à croire que l'individu juge l'autorité avec sa raison ; en revanche, 
des auteurs traditionalistes comme A. Comte ou F. Le Play sont présentés favorablement. Bref, la leçon de J. Locke n'a pas été retenue. Dans sa controverse avec R. Filmer, le célèbre philosophe insistait pourtant sur un point: I'accoutumance n'oblitère jamais la conscience et la liberté de l'individu. Ce n'est pas parce que, étant enfant, I'individu est habitué à obéir à ses parents que cette obéissance est appelée à guider toutes ses actions et ses pensées ultérieures.

\section{La postérité du paradigme politico-psychanalytique}

Dans l'entre-deux guerres, les sciences sociales sont très réceptives à la psychanalyse. En sociologie politique, deux courants se sont inspirés de ce qu'on pourrait appeler le «paradigme politico-psychanalytique »: d'une part, la réflexion sur la culture civique, d'autre part, la théorie sur la persistance des systèmes politiques. Dans les deux cas, si le travail de reformulation du paradigme initial n'est pas négligeable - avec notamment l'abandon formel des concepts psychanalytiques - tout un pan du raisonnement est conservé, puisqu'il s'agit toujours de s'interroger sur les effets politiques induits par I'autorité familiale. L'ouvrage fondateur de Gabriel Almond et Sidney Verba, paru en 1963, vise à répondre à une question simple : pourquoi certains pays sont-ils démocratiques et d'autres non ? L'hypothèse des auteurs est que la démocratie ne se résume pas à de simples mécanismes institutionnels ; pour être viables, les institutions démocratiques doivent être congruentes avec une certaine "culture politique» ou, plus exactement, avec une «culture politique de la participation » (Almond et Verba, 1963). Pour G. Almond et S. Verba, cette culture démocratique s'apprend au cours de l'enfance à partir d'expériences qui ne sont pas directement politiques. En d'autres termes, un individu qui participe aux activités de sa vie quotidienne aura le sentiment qu'il peut peser sur le cours des choses, ce qui va le prédisposer à une bonne participation politique. La famille occupe le premier rôle; elle fait partie des institutions primaires qui assurent l'apprentissage de la culture civique. G. Almond et S. Verba parlent ainsi d'une "connexion entre la démocratie familiale et le comportement démocratique: ceux qui ont eu l'opportunité de participer dans leur famille sont plus nombreux à se sentir compétents politiquement que les autres» (G. Almond et S. Verba, 1963). Les auteurs ne prétendent pas, cependant, tout expliquer par la famille car d'autres institutions participent à la formation de la culture civique : l'école, le groupe des pairs ou encore le lieu de travail. Les réflexions de G. Almond et S. Verba sur les origines culturelles de la démocratie s'inscrivent dans un contexte spécifique. Depuis, elles ont été sérieusement nuancées car le lien entre la culture démocratique et la démocratie paraît plus complexe (Fuchs, 2007).
Mais I'heure était à la comparaison des systèmes et des cultures nationales dans une perspective développementaliste (Badie, 1994). Les États-Unis et la Grande-Bretagne représentaient des démocraties idéales, des idéaux types du civisme vers lesquels devait tendre le reste du monde. La famille américaine était volontiers perçue comme une matrice de la démocratie, par opposition à la famille allemande, plus autoritaire, et favorisant donc une culture de la sujétion (Lane, 1966).

De leur côté, des auteurs comme Robert Hess et, surtout, David Easton, ont proposé d'aller plus loin sur le plan théorique en essayant de décrire ce qui est universel dans le processus de socialisation politique. Ils entendaient comprendre comment un système politique diffuse ses soutiens dans la population, assurant ainsi sa légitimité politique. Dans un article paru en français, en 1963, R. Hess a le plus clairement exposé la version «systémique » du paradigme politico-psychanalytique, laquelle va servir de base théorique à deux équipes de chercheurs : celle de R. Hess et Judith Torney, d'un côté, celle de Jack Dennis et D. Easton, de I'autre (Hess et Torney, 1967 ; Easton et Dennis, 1969). Pour R. Hess, I'acceptation du système politique américain passe par la figure du " président ». L'enfant idéalise le "président», en qui il voit un personnage puissant et invincible, et c'est à partir de là que se forge son attachement au système politique - qui est un attachement "quasi filial ». L'idéalisation du "président» exprime-t-elle la nostalgie freudienne pour le père ? Pour R. Hess, il $\mathrm{n}^{\prime} \mathrm{y}$ a pas à proprement parler de transfert entre le père et le président. La similitude entre ces deux situations s'explique par le fait que l'enfant manifeste un même besoin : celui d'être rassuré en raison de son sentiment de vulnérabilité. Ainsi, il va avoir tendance à idéaliser les autorités qui l'entourent : d'abord son père, puis le président. Le besoin de se sentir protégé pousse donc l'enfant à accorder des qualités de bienveillance au détenteur de l'autorité.

Dans leur ouvrage, David Easton et Jack Dennis ont généralisé cette idée en proposant de décrire le processus d'intégration au système politique par quatre étapes : la politisation, la personnalisation, l'idéalisation et l'institutionnalisation. Au cours de la première phase - la politisation -, l'enfant prend conscience de l'existence d'un monde autre que sa famille. Pour apprivoiser cet univers qui l'entoure, il va s'attacher à ses aspects les plus visibles, c'està-dire les personnalités investies d'une autorité, en l'occurrence le policier et le président (personnalisation). Mais, parallèlement, l'enfant réalise que ce monde est une menace puisque, par définition, il se sent vulnérable. Pour se rassurer, il va doter les personnalités d'une profonde bienveillance à son égard, au point de les considérer comme une sorte de «père idéal » : c'est la phase d'idéalisation. Par la 
suite, I'enfant étendra ses premiers sentiments - qui sont donc d'abord des relations avec des personnes - à l'ensemble des institutions du système politique, et le prestige des institutions prend alors la succession des personnes (phase d'institutionnalisation). Au terme de ce processus, le lien qui rattache l'individu au système devient un lien de confiance et d'affection - et le citoyen est maintenant pleinement intégré. À travers ce modèle théorique, D. Easton et J. Dennis ont envisagé d'identifier les mécanismes universels de la socialisation politique. Mais leur réflexion apparaît, là encore, très marquée par le contexte: celui d'une Amérique en pleine prospérité économique et encore ignorante des conflits à venir.

\section{La famille comme lieu de formation des opinions}

Cependant, un nouveau regard commence déjà à être porté sur la famille, qui met davantage à I'honneur une problématique fondée sur la transmission explicite des opinions politiques. Sous I'influence du béhaviorisme, qui entend justement tourner la page du freudisme, la famille cesse d'être conçue comme un lieu de "production » du politique pour devenir un lieu de "reproduction ». On ne met plus en avant le rôle des relations entre les parents et les enfants dans la structuration du rapport à l'autorité; il s'agit de s'intéresser au transfert direct des opinions politiques d'une génération à la suivante.

\section{La reformulation de Lazarsfeld}

La première étape de cette reformulation se trouve certainement dans l'ouvrage de Paul F. Lazarsfeld, Bernard Berelson et Hazel Gaudet, The People's choice (Lazarsfeld et al., 1948). P. F. Lazarsfeld a lui-même été un compagnon de route de l'école de Francfort avant de se détacher du marxisme et de la psychanalyse. À partir d'un panel d'électeurs interrogés pendant et après l'élection présidentielle de 1940, les auteurs soutiennent une thèse originale : "les gens votent en groupe ». Le groupe est formé d'individus qui présentent les mêmes caractéristiques; il rassemble des gens qui, parce qu'ils travaillent ou vivent ensemble, vont vraisemblablement voter pour le même candidat. Non seulement le groupe est donc "politiquement homogène » mais, de plus, lorsque l'un de ses membres entend voter différemment, il est soumis à des «pressions croisées » qui rétablissent I'homogénéité. Dans ce processus d'homogénéisation politique, la famille occupe une place primordiale. Dans la famille, les conditions de vie sont, en effet, similaires et les contacts mutuels fréquents. Au début de la campagne électorale, il peut y avoir des désaccords ou des incertitudes entre les choix des membres de la famille.
Mais la famille sait créer un " climat d'influence politique». Elle exerce une pression qui assure toujours un changement en faveur du parti qui a sa préférence. C'est de cette façon que peut être assurée la paix à l'intérieur des familles - une paix que tout le monde recherche car elle est confortable sur le plan psychologique. Avec P. Lazarsfeld, les comportements politiques peuvent donc être étudiés sans recourir à l'hypothèse psychanalytique d'une structuration de la personnalité par les relations familiales ou par la nostalgie du père. Mais alors que P. Lazarsfeld ne raisonnait qu'en termes de contraintes, les tenants du béhaviorisme vont vouloir aller plus loin en appliquant à la famille les théories de l'apprentissage et du conditionnement.

\section{La paradigme de Michigan et la transmission des opinions}

À ce titre, l'étape décisive est le "paradigme de Michigan ». Un auteur se révèle ici fondamental: Herbert Hyman, souvent considéré comme l'inventeur de l'expression de "socialisation politique", ce qui n'est pas tout à fait exact (Hyman, 1959). Ses idées se retrouveront dans un ouvrage fondateur de la sociologie électorale, The American Voter, publié peu de temps après (Campbell et al., 1964). Avec H. Hyman, il ne s'agit plus de s'interroger sur les contraintes que la famille exerce de manière ponctuelle au cours des campagnes électorales, mais de comprendre la formation durable des attitudes politiques. Vis-à-vis du paradigme politico-psychanalytique, les éléments de continuité sont de deux ordres : d'une part, l'importance cruciale accordée à la famille comme instance universelle de socialisation, d'autre part, la place décisive de l'enfance dans la formation des opinions. En même temps, c'est sur cette question de l'enfance qu'apparaissent les désaccords avec I'approche psychanalytique. La rupture est explicite lorsque H. Hyman évoque les travaux de Theodor Adorno sur les tendances autoritaires de la personnalité. D'après $\mathrm{H}$. Hyman, il est possible de faire l'économie de la théorie selon laquelle les orientations politiques découlent des structures de la personnalité fixées dans l'enfance. À un modèle indirect et latent, utilisant la structure psychique comme intermédiaire entre l'enfance et les choix politiques adultes, H. Hyman oppose un modèle plus directement politique. Par exemple, l'idéologie autoritaire n'est pas le fruit d'une tendance psychique de la personnalité, qui résulte elle-même $d^{\prime}$ un mode de structuration familiale; elle est tout simplement transmise par les parents.

Cette idée d'une transmission directe n'est pas totalement nouvelle. H. Hyman s'appuie sur plusieurs enquêtes dont la plus ancienne, réalisée par Gordon Allport, remonte à l'élection présidentielle de 1928 (Allport, 1929). Toutefois, avant $\mathrm{H}$. Hyman, très peu 
$\mathrm{d}^{\prime}$ auteurs utilisaient les termes de "transmission» ou "d'héritage ». Allport se contente, par exemple, d'évoquer les "accords» ou les "désaccords» avec le père. D'autres auteurs utilisent diverses formules comme la "tendance à être similaire » ou " à suivre » ses parents, ou encore la "conformité » entre les parents et les enfants. Les notions de « transmission » et d' " héritage » ne sont donc apparues qu'à la fin des années 1950, avec le triomphe des problématiques issues de la psychologie du comportement et des théories de l'apprentissage (le béhaviorisme). Robert Lane va plus loin : I'année de la publication du livre de H. Hyman, il évoque la " "loi mendélienne" de la politique : I'héritage des loyautés politiques et des croyances» (Lane, 1959:502).

Sur le plan empirique, la démonstration de la thèse de I'héritage se révèle toutefois plus délicate que prévue. Dans la plupart des enquêtes citées par $\mathrm{H}$. Hyman, les taux de corrélation entre les attitudes des parents et celles de leurs enfants ne sont pas très élevés. Il va néanmoins estimer que la corrélation moyenne est de .50, ce qui, de son propre aveu, est assez modéré. H. Hyman en déduit même que "les parents ne sont que l'un des agents de la socialisation et que leur influence n'est pas si grande " (Hyman, 1959:72). Mais à ses yeux, ce résultat ne met nullement en cause le rôle décisif de la famille. Tout d'abord, il relève qu'aucune enquête n'a rencontré de corrélations négatives, ce qui lui permet d'affirmer que les attitudes politiques des enfants ne se forment pas en opposition avec celles des parents. Ensuite, et surtout, $\mathrm{H}$. Hyman souligne que la corrélation entre parents et enfants est nettement plus élevée dans le cas des proximités envers un parti politique. À ses yeux, ce résultat est le plus décisif. En effet, H. Hyman admet volontiers que la famille ne transmet pas toujours des opinions politiques très précises. Mais le plus important, c'est qu'elle assure la transmission d'une sorte de " grille de lecture » à partir de laquelle l'enfant va pouvoir appréhender les enjeux pour lesquels il n’a pas été préparé.

\section{L'héritage politique en France : la querelle autour du French case}

En France, la réflexion sur la transmission politique a été plus tardive qu'aux États-Unis en raison d'une tradition sociologique longtemps centrée sur la lutte des classes et la reproduction du capital culturel. Ce n'est que depuis les années 1970 que la question de la transmission politique a suscité davantage d'intérêt. Outre le déclin des théories néomarxistes, un élément a encouragé les investigations: la volonté de répondre à deux auteurs, Philipp Converse et Georges Dupeux, qui avaient mis en cause la «qualité » de la transmission politique au sein des familles françaises. Dans un article publié en 1962, P. Converse et G. Dupeux ont, en effet, soutenu que I'instabilité politique chronique de la France (le fameux «French case ») s'explique par la "particularité du processus français de socialisation ", en l'occurrence l'absence de transmission des orientations politiques entre parents et enfants (Converse et Dupeux, 1962 ; Tournier, 2000). Leur démonstration s'appuyait notamment sur un résultat: la faible proportion de Français capables d'indiquer I'affiliation partisane de leurs pères (24\% contre $82 \%$ aux États-Unis), ce qui était pour eux le signe d'une mauvaise transmission familiale.

Déjà, dans un livre paru en 1974, Annick Percheron avait objecté que P. Converse et G. Dupeux se trompaient de cible: en France, les clivages politiques ne passent pas par l'identification à un parti mais par des orientations idéologiques exprimées en termes de gauche et de droite. La faiblesse de la transmission des préférences partisanes découle surtout des particularités du système de partis en France - explication qui rejoint celle avancée par David R. Cameron et Laura Summers sur le rôle des facteurs non familiaux de socialisation politique (Cameron et Summers, 1972). En s'appuyant sur les résultats d'une enquête « appariée » auprès de parents et d'enfants réalisée en 1975, Annick Percheron montre que "contrairement à ce qu'écrivaient Converse et Dupeux il y a près de vingt ans, la France se caractérise, à l'instar des autres pays, par la continuité plutôt que par la discontinuité dans la transmission des choix idéologiques d'une génération à l'autre " (Percheron et Jennings, 1981:120). Autrement dit, I'héritage politique n'est pas une spécificité américaine ; il correspond à une situation qui s'impose dans toutes les cultures car il existe une " universalité des processus et des fonctions de socialisation, par-delà la diversité des contenus culturels » (id :129). Cette conclusion était probablement audacieuse (Westholm et Niemi, 1992). Néanmoins, une nouvelle enquête, réalisée en 1989, est venue confirmer l'importance de la continuité idéologique en France. La comparaison des deux enquêtes de 1975 et 1989 montre $\mathrm{qu}^{\prime}$ « en 1975, 34 \% des 16-18 ans reproduisaient exactement les positions de leurs parents sur l'axe gauche-droite; en 1989, ils sont $49 \%$ à le faire (soit une augmentation de 15 points de pourcentage) » (Percheron, 1989:80). La transmission familiale ne s'est donc pas affaiblie; peut-être même y a-t-il une "augmentation du nombre des héritiers» (Percheron, 1989 :87).

\section{Le temps des critiques}

Paradoxalement, c'est au moment où les chercheurs français s'efforcent de confirmer la thèse de l'héritage politique que celle-ci fait l'objet d'une remise en 
cause. À partir de la fin des années 1960, les études de socialisation politique commencent, en effet, à subir de sérieuses critiques (Connell, 1987). Il est vrai que le contexte ne joue plus en leur faveur. Avec la multiplication des revendications sociales (jeunes, féministes, minorités ethniques, etc.), I'hypothèse d'un changement radical entre les jeunes générations et les anciennes générations gagne du crédit. Des auteurs tels que Margaret Mead aux États-Unis [1979(1969)] ou Georges Mendel en France (1971) proposent des théories sur le "fossé » ou le «conflit » des générations (Attias-Donfut, 1991), au risque de céder à un excès inverse : après le temps du "tout famille ", voici venu le temps de la rupture entre les générations.

\section{Continuité... ou rupture entre les générations?}

Ce regain d'intérêt pour la notion de " génération » a le mérite de mettre au premier plan la question du changement - question largement délaissée jusquelà. En Angleterre, David Butler et Donald Stokes - défenseurs du paradigme de Michigan - ont recours aux effets de génération pour expliquer la croissance du parti travailliste et la baisse du lien entre l'appartenance à la classe ouvrière et le vote travailliste (Butler et Stokes, 1969). Une conclusion similaire est tirée par Paul Abramson pour les ÉtatsUnis : entre 1948 et 1968, la relation entre l'appartenance sociale et le vote a diminué en raison d'un " changement générationnel» (Abramson, 1974). Critiquant le paradigme de Michigan, P. Abramson soutient également qu'il existe un déclin de l'identification partisane dans les générations de l'aprèsguerre, invalidant ainsi la thèse de la transmission familiale (Abramson, 1989). C'est également à partir des années 1970 que Ronald Inglehart, coauteur de plusieurs études avec P. Abramson, développe la thèse de la « révolution silencieuse » en lien avec la montée des valeurs "post-matérialistes " dans les générations nées après la guerre (Inglehart, 1971 ; Inglehart, 1993 ; Abramson et Inglehart, 1993). Les études françaises confirment l'existence d'importants effets de génération dans le domaine des valeurs morales, avec notamment le déclin de la religion catholique et la montée du libéralisme moral (Percheron, 1982 ; Drouin, 1995). Dans ces conditions, la thèse de la "reproduction familiale » ne peut plus bénéficier du même crédit qu'auparavant : comment croire que les enfants reproduisent les idées de leurs parents si les sociétés se transforment profondément ? Un retour sur les certitudes initiales est devenu nécessaire.

\section{La délicate question de l'enfance}

Assez logiquement, la discussion s'est d'abord portée sur l'enfance. Sous la double influence de la psychanalyse et du béhaviorisme, un postulat a longtemps été admis sans discussion : I'enfant est un être passif et les apprentissages politiques se jouent très tôt (Searing et al., 1973). D'où la focalisation des recherches sur l'enfance et le peu d'intérêt pour la socialisation politique des adultes (Sigel, 1989). Certes, les premiers choix politiques ne commencent pas avec l'âge de la majorité électorale, ce qui invalide le mythe de I' «innocence politique » des enfants (Percheron, 1978). Assez tôt, les enfants sont capables d'identifier les fonctions et les rôles politiques, d'afficher des préférences pour des personnalités ou des mouvements politiques, voire de manier des raisonnements politiques sophistiqués. Cela étant, plusieurs questions paraissent avoir été négligées. Sur la méthodologie tout d'abord: à partir de quel âge est-il possible d'utiliser des questionnaires fermés, en sachant que les enfants manifestent un fort désir de coopérer et une tendance à I'acquiescement (Kolson et Green, 1970) ? Sur le fond ensuite : que deviennent les apprentissages de I'enfance? Sont-ils définitifs? En s'appuyant sur I'exemple de la France, Fred Greenstein se demande ironiquement pourquoi, en dépit d'une apparente "tranquillité politique», les jeunes Français ont " activement participé aux événements de Mai 68 » (Greenstein, 1970:973-974). Quelques années plus tard, il renouvelle sa question en critiquant les théories qui ont fait de l'idéalisation des figures d'autorité la clé de voûte de la socialisation politique : "certains adolescents qui idéalisaient allègrement Eisenhower et Kennedy lors des premières enquêtes ont tout aussi bien pu être les leaders des protestations contre le "système" quelques années plus tard»(Greenstein, 1975:1373).

Pour des auteurs comme David Marsh ou Donald Searing, la place accordée à l'enfance relève moins d'une démarche scientifique que d'une «croyance » qui n’a jamais été sérieusement testée empiriquement (Marsh, 1971 ; Searing et al., 1973). Le manque d'études longitudinales est vivement dénoncé. Or, en interrogeant un millier d'enfants de 9 à 15 ans à trois reprises au cours de l'année 1968-1969, la Canadienne Pauline Marie Vaillancourt montre que I'instabilité des réponses est très forte, y compris sur la religion (seulement $72 \%$ des enfants ont fait le même choix entre catholique, protestant, juif et sans religion) ou sur l'identification à un parti politique $(50 \%$ des enfants ont donné la même réponse). Cette instabilité ne concerne pas seulement les items isolés, mais aussi les indices, ce qui conduit l'auteur à penser que les enfants n'ont encore que des «formes embryonnaires d'attitudes» (Vaillancourt, 1973). En France, Anne Muxel a validé ce résultat à partir d'un panel de près de deux mille jeunes interrogés pour la première fois en novembre 1986 à l'âge de 17-19 ans, puis à cinq reprises jusqu'en 1997. Les résultats montrent qu'il existe une forte variabilité des réponses. Entre la première vague et la dernière, à peine $18 \%$ des jeunes sont restés fidèles au même classement sur 
une échelle gauche-droite tandis que seulement $15 \%$ ont déclaré la même proximité partisane (Muxel, 2001).

Cette instabilité des opinions soulève de nombreuses questions. Les opinions des enfants renseignentelles vraiment sur les opinions et les choix qui seront effectuées à l'âge adulte? D. Searing et ses collègues avancent un argument redoutable pour le paradigme de Michigan : même si les préférences partisanes sont plutôt bien transmises entre les générations, celles-ci se révèlent peu prédictives des opinions émises par les adultes sur les grands enjeux de société, ce qui remet en cause le «principe de structuration» (structuring principle), c'est-à-dire l'idée selon laquelle les « orientations de base acquises dans l'enfance structurent la perception des enjeux à venir » (Searing et al., 1973:415). Un argument similaire pourrait être utilisé en France au sujet de l'échelle gauchedroite. Par ailleurs, les adultes sont eux-mêmes loin d'avoir des opinions stables. D'une élection à I'autre, leurs choix peuvent fluctuer. Même dans le domaine des valeurs, les études par cohortes indiquent que les changements ne concernent pas que les jeunes générations mais touchent souvent I'ensemble des tranches d'âge (Tournier, 2004). Certes, les opinions sont plus fluctuantes chez les adolescents que chez les adultes (Alwin et Krosnick, 1991) mais elles ne sont jamais totalement figées ; et si elles ont tendance à se cristalliser, c'est plutôt vers la fin de l'adolescence, voire au début de I'âge adulte (Vollerbergh et al., 2001), donc à un moment où les jeunes sont déjà en mesure de relativiser les idées parentales.

Comment admettre que les expériences vécues dans l'enfance éclairent les comportements politiques des adultes si les opinions de ces derniers sont elles-mêmes fluctuantes, peu prévisibles et tributaires des enjeux du moment? Les spécialistes de la socialisation politique ont peut-être adhéré trop vite à une vision sommaire de la nature humaine, qui consiste à partir de l'idée que le présent s'explique systématiquement par le passé, donc que l'adulte n'est pas un être réellement autonome, capable de s'extraire de ses origines et de ses déterminations. Cette vision correspond à ce que l'écrivain Milan Kundera a appelé le "réalisme psychologique » et qu'il définit de la façon suivante: "deux siècles de réalisme psychologique ont créé quelques normes quasi inviolables : 1. il faut donner le maximum d'informations sur un personnage, sur son apparence physique, sur sa façon de parler et de se comporter ; 2. il faut faire connaître le passé d'un personnage, car c'est là que se trouvent toutes les motivations de son comportement présent» (Kundera, 1986:51). Ce modèle du réalisme psychologique établit une causalité temporelle qui voit dans la vie de chacun le fruit d'une sédimentation par couches successives. Or, I'individu entretient un rapport complexe avec son passé. La mémoire fait l'objet d'une construction et d'une reconstruction permanentes au gré des circonstances et des enjeux du moment, car les événements, qu'ils soient personnels ou politiques, jouent également un rôle important dans la formation et la transformation des opinions (Stoker et Jennings, 1995 ; Sears et Valentino, 1997).

\section{L'influence de la famille a-t-elle été exagérée?}

Concernant le rôle de la famille dans la transmission politique, deux grandes critiques ont été émises. La première porte sur les aspects méthodologiques. Les études ayant insisté sur la continuité entre parents et enfants ont souvent utilisé des effectifs modestes, sans qu'une réelle attention ne soit portée à la représentativité des échantillons, ce qui a manifestement conduit à surreprésenter les familles où les relations domestiques se passent bien (Connell, 1972). Par ailleurs, Richard Niemi a critiqué les enquêtes qui se sont contentées d'interroger des jeunes pour avoir des informations sur leurs parents (Niemi, 1973). La seconde critique porte sur I'ampleur de la transmission. En s'appuyant sur une enquête de 1965 interrogeant directement des parents et leurs enfants, Kent Jennings et R. Niemi ont revu à la baisse l'importance de la continuité entre parents et enfants, les corrélations étant au mieux modérées (Jennings et Niemi, 1968 ; Niemi et al., 1978). Même pour les préférences partisanes, élément clé du paradigme de Michigan, K. Jennings et R. Niemi obtiennent une corrélation inférieure à .50, pratiquement deux fois moins élevée que celle avancée par H. Hyman, ce qui les conduit à conclure qu'un jeune n'est pas « une simple copie carbone de ses parents " (Jennings et Niemi, 1968:183). Cette enquête de 1965 a été renouvelée auprès des mêmes personnes à deux reprises, en 1973 et 1982. Les résultats ont confirmé les tendances observées en 1965, en y ajoutant un effet du cycle de vie, car les choix politiques des parents ont surtout une influence "quand les enfants sont à la maison» (Beck et Jennings, 1991 ; voir aussi Vollerbergh et al, 2001).

Pour éviter tout malentendu, K. Jennings et R. Niemi prennent soin de préciser que la faiblesse des corrélations entre parents et enfants ne signifie pas que les enfants se révoltent contre leurs parents. Les divergences peuvent tenir à plusieurs raisons : 1. les jeunes optent consciemment pour des valeurs différentes de celles de leurs parents ; 2 . les parents ont parfois des idées politiques ambiguës, peu précises, ce qui ouvre la porte à d'autres agents de socialisation politique; 3 . certaines opinions sont parfois instables, ou du moins ne bénéficient pas d'une position centrale dans les systèmes de croyance ; 4 . il faut tout simplement tenir compte 
des effets du cycle de vie. Concernant ce dernier point, il a été vérifié, par exemple, que la politisation évolue en fonction des étapes de la vie car il existe " un calendrier social réglant les rapports à la politique »(Denni, 1995:27). La moindre politisation des jeunes est moins le fruit d'un effet de génération que d'un effet du cycle de vie; elle est sensible à I'insertion sociale et professionnelle, tout en étant fortement liée à la conjoncture politique. D'autres enquêtes ont confirmé les conclusions de K. Jennings et R. Niemi, notamment une enquête lancée à Los Angeles en 1973 qui a interrogé simultanément trois générations (grands-parents, parents et enfants). Les résultats montrent que les enfants ont une idée très imprécise des opinions politiques de leurs parents (Acock et Bengston, 1980 ; Miller et Glass, 1989). Surtout, cette enquête permet de vérifier que les corrélations entre parents et enfants sont faibles mais qu'elles sont plus élevées si on remplace les réponses des parents par les indications fournies par les enfants sur leurs propres parents, comme I'avait d'ailleurs montré R. Niemi (Niemi, 1973). Autrement dit, les enfants sont moins proches de leurs parents que de l'image qu'ils s'en font. Ce résultat permet d'envisager la transmission non pas comme un processus objectif et passif, mais comme un processus interactif et subjectif, dans lequel l'enfant intervient pleinement.

\section{Renouveler les approches}

Avec les critiques des années 1970, un tournant s'est produit. Désormais, la transmission politique ne va plus de soi ; elle n'est plus considérée comme une vérité qui ne demande qu'à être confirmée. La mise en cause d'une tendance "naturaliste » qui imprégnait jusque-là bon nombre d'études oblige à porter un autre regard sur la transmission politique et, du même coup, sur l'individu lui-même. Un renouvellement théorique se fait sentir : si la similitude ne va plus de soi, si elle ne peut plus être considérée comme une production spontanée de la famille, c'est donc qu'il faut l'expliquer, l'interpréter.

\section{Comprendre les facteurs de la continuité}

Finalement, la question n'est plus vraiment de savoir s'il existe des similitudes entre parents et enfants. Ces similitudes existent, c'est incontestable. Elles sont même probablement plus fortes que I'ont pensé Jennings et Niemi - c'est du moins ce que soutient Russel J. Dalton qui, à partir de leurs données, a montré que les corrélations dans divers domaines (confiance, racisme, civisme, etc.) sont plus élevées si on remplace les items simples par des indices composites (Dalton, 1980) - et elles ont aussi tendance à perdurer après le départ des enfants du domicile familial, contrairement à ce que pensaient Ulrich Beck et K. Jennings (Vollerbergh et al., 2001). Mais l'enjeu est désormais de savoir quelle signification donner à ces similitudes: qu'est-ce qui les rend possibles ? quels sont les mécanismes par lesquels celles-ci se réalisent? Une réflexion de ce type avait déjà été esquissée par $\mathrm{K}$. Jennings et R. Niemi dans une optique essentiellement négative (Jennings et Niemi, 1968). Pour ces chercheurs, il s'agissait de contester le rôle de deux facteurs habituellement utilisés pour expliquer la transmission: le sexe et la structure de l'autorité. Les résultats obtenus en 1965 les conduisaient ainsi à rejeter l'impact des "rôles sexuels » (les corrélations n'évoluent pas si l'on distingue le père et la mère d'un côté, le fils et la fille de l'autre) et, surtout, le type de relations entre parents et enfants (comme le sentiment de proximité ou le degré d'autonomie).

Dans un article publié en 1974, Kent Tedin a proposé une démarche plus positive en essayant d'identifier les conditions de la continuité politique (Tedin, 1974). K. Tedin pose, en effet, que les parents disposent d'un "potentiel inhérent pour réussir la transmission» (p. 1592) mais que la réalisation de ce potentiel est soumis à deux conditions : d'une part, l'importance que les parents accordent à un enjeu politique et, d'autre part, l'exactitude des perceptions que les enfants se font des attitudes de leurs parents. Malgré les limites de sa démonstration (l'échantillon est assez faible), la démarche de K. Tedin n'en reste pas moins novatrice. En France, A. Percheron s'en est inspirée. Pour elle, plusieurs conditions favorisent la transmission idéologique au sein de la famille: 1 . la capacité des parents à transmettre un message (qui dépend du niveau de diplôme ou de politisation) ; 2 . la visibilité des choix des parents ; 3 . I'homogénéité du milieu de socialisation, notamment l'homogénéité entre les deux parents (Percheron, 1985).

Le bien-fondé de ce cadre théorique a été vérifié (Tournier, 1998). À partir d'une enquête menée auprès de parents et de lycéens dans le département de l'Isère, deux conclusions se sont dégagées. Tout d'abord, il convient de préciser de quoi on parle lorsqu'on parle de «transmission ». Traditionnellement, deux options sont envisagées : soit les jeunes reproduisent totalement les idées de leurs parents, soit ils s'en éloignent radicalement. On oppose abruptement, d'un côté, les héritiers, de l'autre, les jeunes en rupture. La réalité semble plus complexe, les frontières plus floues. Entre 15 ans et 18 ans, les jeunes qui reproduisent étroitement les idées de leurs parents, comme ceux qui s'en éloignent fortement, semblent minoritaires. La plupart des jeunes se trouvent dans des situations intermédiaires: ils partagent certaines idées parentales, s'en démarquent sur d'autres ; ils construisent leur personnalité au gré de leur parcours et de leurs 
expériences sociales. On peut penser que ces prises de distance à l'égard des parents sont facilitées par le contexte actuel des sociétés modernes, où l'indépendance et l'esprit critique des enfants sont fortement valorisés sans que soient pour autant remises en cause les solidarités familiales.

La seconde conclusion est que les convergences entre parents et enfants ne sont nullement automatiques ou spontanées. Comme le laissaient entendre $\mathrm{K}$. Tedin et A. Percheron, elles émergent dans un cadre familial particulier et apparaissent liées à une série de conditions qui sont spécifiquement politiques : le degré de politisation des parents, le degré d'homogénéité et de structuration de leurs choix, la fréquence des échanges politiques entre parents et enfants, autant de facteurs qui mettent en jeu une capacité à exposer des choix, à les argumenter, donc à les rendre légitimes - condition de leur appropriation par les enfants.

\section{L'influence directe des parents : une vision trop simple?}

Au-delà, la question de la signification de l'influence est également posée. On retrouve ici un problème classique en sciences sociales: le passage de la corrélation à la causalité. Les similitudes entre parents et enfants ont toujours été vues comme le produit d'une transmission unidirectionnelle. Mais I'influence n'est-elle pas aussi à double sens? Comme le suggérait déjà $M$. Mead en analysant les sociétés qui connaissent les changements rapides, les jeunes ne sont-ils pas susceptibles d'influencer leurs parents - comme c'est par exemple le cas dans les familles issues de l'immigration?

Une enquête auprès de parents et de jeunes allemands âgés de 11 ans à 17 ans réalisée en deux vagues à un an d'intervalle montre que les opinions des enfants en début d'enquête prédisent assez bien l'évolution des opinions parentales sur certains sujets comme l'utilisation des nouvelles technologies, mais aussi le degré d'adhésion aux valeurs traditionnelles ou religieuses $-y$ compris dans les familles qui ne sont pas forcément les plus ouvertes ou les plus conciliantes (Pinquart et Silbereisen, 2004). Ces résultats sont confirmés par d'autres enquêtes (Glass et al., 1986), mais infirmés par d'autres (Vollerbergh et al., 2001). Par ailleurs, les similitudes entre parents et enfants ne doivent-elles pas être envisagées dans un cadre plus large? Autrement dit, ne découlent-elles pas du fait que les parents et leurs enfants ont une vie sociale en grande partie commune? Cette question a été posée par Judith Harris dans un ouvrage volontairement provocateur (Harris, 1999). Selon elle, pour des raisons culturelles, on exagère considérablement l'importance des parents alors que, en réalité, l'essentiel de la socialisation se fait par le groupe des pairs. L'hypothèse est crédible mais elle mérite d'être précisée. Les fréquentations des enfants dépendent aussi, pour partie, des parents euxmêmes. Le raisonnement doit donc être élargi. II s'agit alors de considérer que, en raison de leurs caractéristiques sociales et professionnelles, les parents définissent un cadre de socialisation (statut social, réseaux de sociabilité, cursus scolaire, pratiques culturelles, etc.), qui délimite le champ des expériences sociales, qu'il s'agisse de leurs propres expériences ou de celles de leurs enfants. Une influence indirecte des parents coexiste ainsi avec une influence directe (Glass et al., 1986; Vollerbergh et al., 2001). Par exemple, il a été démontré que la participation politique à l'âge adulte (mesurée par l'implication dans diverses activités politiques) relève d'un ensemble complexe de facteurs parmi lesquels la contribution directe des parents est moins importante que leur contribution indirecte (Beck et Jennings, 1982).

D'une certaine façon, cette réflexion permet de faire le lien avec la problématique des cultures politiques, remise à I'honneur depuis quelques années grâce aux programmes de recherches internationaux (Inglehart, 1988). Les comparaisons internationales montrent, en effet, que les sociétés - y compris lorsqu'elles sont comparables par leur niveau de richesse - n'ont pas les mêmes valeurs culturelles et politiques, et que ces différences se maintiennent au fil du temps en dépit de certaines évolutions (Inglehart, 2000). Cette relative stabilité collective ne peut s'expliquer par la seule transmission au sein des familles. Une forte variabilité interfamiliale n'est donc pas incompatible avec le maintien des spécificités culturelles, ce qui confirme que la socialisation politique ne se réduit pas à la famille. C'est rappeler que la socialisation politique s'inscrit dans un contexte plus global et qu'elle est largement conditionnée par les caractéristiques d'un pays, par ses institutions, sa mémoire, ses clivages - ce que Émile Durkheim avait jadis appelé sa « conscience collective».

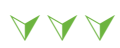

\section{Conclusion}

Toutes les interrogations sur la famille sont loin d'avoir été levées. Le seront-elles un jour? Les critiques qui ont été émises depuis les années 1970 ont nettement affaibli l'intérêt pour les études de socialisation politique, au point que ce champ de recherche a pu paraître "dans une impasse " (Percheron, 1981). Il est vrai que les premières études ont été trop naïves, trop sûres d'elles-mêmes. Une question aussi simple que «qu'est-ce que la famille? »n'a jamais été réellement posée et prise en compte dans les enquêtes, alors même que la 
diversification des structures familiales la rend plus incontournable que jamais. Malgré tout, les critiques ont également permis de faire entrer la recherche dans une phase de maturité. Les affirmations rapides et catégoriques d'autrefois ne sont plus de mise. Aujourd'hui, les exigences sont plus fortes; les enquêtes sont tenues de proposer des problématiques plus subtiles, de mettre en place des protocoles d'observation plus complexes. L'objectif est moins de confirmer ou de contester l'importance de la famille que de comprendre les ressorts et les limites de son intervention. De ce fait, un mouvement de balancier s'est produit: alors que les premières études brandissaient des conclusions catégoriques tout en étant faiblement documentées, les études récentes sont plus robustes mais leurs conclusions plus prudentes. Plus l'observation gagne en précision, plus la réalité apparaît complexe, par conséquent difficile à condenser en une conclusion définitive.

En particulier, on ne trouve plus guère d'auteurs pour soutenir que les opinions ou les comportements politiques sont déterminés par le style édu- catif des parents ou le type d'autorité exercé dans la famille. Les études qui ont essayé de faire le lien entre les structures familiales et le développement des opinions politiques n'ont pas débouché sur des résultats probants (Dolan, 1995). Mais cette piste devrait probablement être reprise et approfondie, notamment dans le cadre des réflexions sur le capital social. Les comparaisons internationales montrent que la famille recouvre des réalités très différentes selon les sociétés (qu'il s'agisse des perceptions de la famille, des formes de vie familiale, de l'âge du départ du domicile) mais l'on sait finalement peu de choses sur les liens qui peuvent exister entre ces diverses dimensions de la famille et le développement des attitudes politiques et civiques. Plus généralement, on peut souhaiter que les futures recherches s'attachent à mieux documenter les différentes sources d'influence qui se manifestent parallèlement à la famille (école, médias, pairs, contexte socioculturel), tout en essayant de suivre l'évolution des opinions dans la durée, ce qui éviterait de trop donner le sentiment que les opinions sont comme figées de manière précoce.

\section{\begin{tabular}{l|l} 
& \\
Références & bibliographiques
\end{tabular}}

- Abramson P. R., 1989, Générations et changement politique aux États-Unis, in Générations et politiques (sous la dir. de Crête J. et Favre P.), Presses Universitaires de Laval, Economica, collection Politique comparée:89-149.

- Abramson P. R., 1974, Generational change in American electoral behavior, American Political Science Review, vol. 68, $\mathrm{n}^{\circ}$ 1:93-105.

- Abramson P. R. et Inglehart R., 1993, Generational replacement and value change in eight West European societies, British Journal of Political Science, vol. 22, $\mathrm{n}^{\circ} 2: 183-228$.

- Acock A. C. et Bengtson V. L., 1980, Socialization and attribution processes: actual versus perceived similarity among parents and youth, Journal of Marriage and the Family, vol. 42, n 3:501-515.

- Adorno T.-W., Frenkel-Brunswik E, Levinson D.-J. et Nevitt Sanford R., 1950, The authoritarian personality, New York, Harper \& Brothers.

- Allport G.-W., 1929, The composition of political attitudes, The American Journal of Sociology, n०35:220-238.

- Almond G.-A. et Verba S., 1963, The civic culture. Political attitudes and democracy in five nations, Princeton, New Jersey, Princeton University Press.

- Alwin D. F. et Krosnick J. A., 1991, Aging, cohorts, and the stability of sociopolitical orientations over the life span, American Journal of Sociology, vol. 97, $\mathrm{n}^{\circ}$ 1:169-195.

- Attias-Donfut C., 1991, Générations et âges de la vie, Paris, Presses universitaires de France, collection «Que sais-je?».

- Badie B., 1994, Le développement politique, Paris, Economica, $1^{\text {re }}$ édition 1980.

- Beck P. A. et Jennings M. K., 1991, Family traditions, political periods and the development of partisan orientations, The Journal of Politics, vol. 53, $\mathrm{n}^{\circ}$ 3:742-763.

- Beck P. A. et Jennings M. K. 1982, Pathways to participation, American Political Science Review, vol. 76, n 1:94-108. 
- Borgetto M., 1983, Métaphore de la famille et idéologies, in Le droit non civil de la famille (sous la dir. de Carbonnier J.), Paris, Presses universitaires de France:1-21.

- Bossuet J.-B., 1864 (1709), Politique tirée des propres paroles de l'Écriture sainte, in CEuvres complètes, tome XIII, J.-B. Pélagaud.

- Bréchon P., 1976, La famille, idées traditionnelles, idées nouvelles, Le Centurion, Socioguides.

- Butler D. et Stokes D., 1969, Political change in Britain: Forces shaping electoral choice, London, MacMillan.

- Cameron D. R. et Summers L., 1972, Non-family agents of political socialization: A reassessment of Converse and Dupeux, Canadian Journal of Political Science, vol. 5, n 3:418-432.

- Campbell A., Converse P-E., Miller W.-E. et Stokes D-E., 1964, The american voter, an abridgment, Survey Research Center, University of Michigan, John Wiley \& Sons Inc., $1^{\text {re }}$ édition 1960.

- Comte A., 1975 (1842), Physique sociale : cours de philosophie positive, Leçons 46 à 50, Hermann, Éditeurs des Sciences et des Arts.

- Connell R.W., 1987, Why the "political socialization» paradigm failed and what should replace it? International Political Science Review, vol. 8, n 3:215-223.

- Connell R.W., 1972, Political socialization and the famille: The evidence reexamined, Public Opinion Quarterly, vol. 36, n³:323-333.

- Converse P. et Dupeux G., 1962, Politization of the electorate in France and in the United States, Public Opinion Quaterly, vol. 26, n 1:1-23.

- Dalton R., 1980, Reassessing parental socialization: Indicator unreliability versus generational transfert, American Political Science Review, vol. 74, n² 2:421-431.

- Denni B., 1995, Age, attitudes et comportements politiques, Gérontologie et société, n 74:20-33.

- Dolan K., 1995, Attitudes, behaviors and the influence of the family: A reexamination of the role of family structure, Political Behavior, vol. 17, $\mathrm{n}^{\circ}$ 3:251-264.

- Drouin V., 1995, Enquêtes sur les générations et la politique, 1958-1995, Paris, L'Harmattan, collection Logiques politiques.

- Easton D. et Dennis J., 1969, Children in the political system. Origins of political legitimacy, New York, Mc Graw-Hill Book Company.

- Filmer R., 1991 (1640) Patriarcha, ou du pouvoir naturel des rois, Paris, L'Harmattan, collection Logiques sociales.

- Fuchs D., 2007, The paradigm of political culture, in Oxford Handbook of Political Science (sous la dir. de Dalton R. J. et Klingemann H.-D.), Oxford University Press:161-184.

- Glass J., Bengston V. et Duham C., 1986, Attitude similarity in three-generation families: Socialization, status inheritance, or reciprocal influence?, American Sociological Review, vol. 51, n 5:685-698.

- Greenstein F. I., 1970, A note on the ambiguity of " political socialization »: definitions, criticisms, and strategies of inquiry, The Journal of Politics, vol. 32, $n^{\circ} 4: 969-978$.

- Greenstein F. I., 1975, The benevolent leader revisited : children's images of political leaders in three democracies, American Political Science Review, vol. 69, n 4:1371-1398.

- Harris J. R., 1999, Pourquoi nos enfants deviennent ce qu'ils sont ?, Paris, Robert Laffont.

- Hess R. D., 1963, La socialisation des attitudes à l'égard de l'autorité politique : comparaison entre quelques pays, Revue internationale des sciences sociales, vol. 15, $\mathrm{n}^{\circ}$ 4:574-593.

- Hess R.D. et Torney J.V., 1967, The development of Political attitudes in Children, Chicago, Aldine Publishing Company.

- Horkheimer M., 1974, Théorie traditionnelle et théorie critique, Paris, Gallimard, NRF, collection Les essais.

- Hyman H., 1959, Political socialization: A study in the psychology of political behavior, Glencoe, Illinois, The Free Press.

- Inglehart R., 2000, Modernization, cultural change, and the persistence of traditional values, American Sociological Review, vol. 65, $\mathrm{n}^{\circ}$ 1:19-51. 
- Inglehart R., 1993[1990], La transition culturelle dans les sociétés industrielles avancées, Economica, Politique comparée.

- Inglehart R., 1988, The renaissance of political culture, American Political Science Review, vol. 82, $\mathrm{n}^{\circ} 4: 1203-1230$.

- Inglehart R., 1971, The silent revolution in Europe : intergenerational change in post-industrial societies, American Political Science Review, vol. 65, n² 4:991-1017.

- Jennings M. K. et Niemi R. G., 1968, The transmission of political values from parent to child, American Political Science Review, vol. 62, $\mathrm{n}^{\circ}$ 1:169-184.

- Kolson K. L. et Green J. J., 1970, Response set bias and political socialization research, Social Science Quarterly, vol. 51, n 3:527-538.

- Kuhn T., 1983 (1962), La structure des révolutions scientifiques, Paris, Flammarion.

- Kundera M., 1986, L'art du roman, Gallimard, NRF.

- Lane R. E., 1966, La maturation politique de I'adolescent aux États-Unis et en Allemagne, Revue française de sociologie, $\mathrm{n}^{\circ}$ 7:598-618.

- Lane R. E., 1959, Fathers and sons: Foundations of political belief, American Sociological Review, $n^{\circ} 24: 502-511$.

- Lazarsfeld P., Berelson B. et Gaudet H., 1948[1944], The People's choice. How voter makes up his mind in a presidential campaign, New York, Columbia University Press.

- Le Play F., 1989 (1879), La méthode sociale, présentation d'Antoine Savoye, Méridiens Klincksieck, Analyse institutionnelle.

- Locke J., 1992 (1690), Traité du gouvernement civil, Paris, Flammarion.

- Maistre J. (de), 1989[1793-1798], Ecrits sur la Révolution, Presses universitaires de France, Quadrige.

- Marsh D., 1971, Political socialization: the implicit assumptions questionned, British Journal of Political Science, 1:453-465.

- Mead M., 1979 (1969), Le fossé des générations : les nouvelles relations entre les générations dans les années 1970, Paris, Denoël-Gonthier.

- Mendel G., 1971 [1969], La crise des générations. Étude sociopsychanalytique, Petite Bibliothèque Payot.

- Miller R. B. et Glass J., 1989, Parent-child attitude similarity across the life course, Journal of Marriage and the Family, vol. 51, n ${ }^{\circ}$ 4:991-997.

- Muxel A., 2001, Les choix politiques des jeunes à l'épreuve du temps : une enquête longitudinale, Revue française de science politique, vol. 51, $\mathrm{n}^{\circ}$ 3:409-430.

- Niemi R. G., 1973, Collecting information about the family: a problem in survey methodology, in Socialization to politics: A reader (sous la dir. de Dennis J.), John Wiley \& Sons:464-490.

- Niemi R. G., Ross R. D. et Alexander J., 1978, The similarity of political values of parents and college-age youths, Public Opinion Quarterly, vol. 42, n 4:503-520.

- Percheron A., 1989, Peut-on encore parler d'héritage politique en 1989 ?, in Pour Lavau. Idéologies, partis politiques et groupes sociaux (sous la dir. de Mény Y.), Paris, Presses de la Fondation nationale des sciences politiques:71-88.

- Percheron A., 1985, La socialisation politique. Défense et illustration, in Traité de science politique, "L'Action politique » (sous la dir. de Grawitz M. et Leca J.), Paris, PUF:165-235.

- Percheron A., 1982, Préférences idéologiques et morale quotidienne d'une génération à l'autre, Revue française de science politique, vol. 32, $\mathrm{n}^{\circ} 2: 185-209$.

- Percheron A. et Jennings M.-K., 1981, Political continuities in French families. A new perspectives on an old controversy, Comparative Politics, vol. 3, n 4:421-436, texte partiellement traduit en français dans Percheron A., 1993, Le mythe de l'exceptionnalité française, in La socialisation politique (sous la dir. de Percheron A.), textes réunis par Nonna Mayer et Anne Muxel, Armand Colin, Collection U:119-129.

- Percheron A., 1981, Les études américaines sur les phénomènes de socialisation politique dans I'impasse? Chronique d'un domaine de recherche, L'Année sociologique, n 31:69-96. 
- Percheron A., 1978, Les 10-16 ans et la politique, Paris, Presses de la Fondation nationale des sciences politiques.

- Percheron A., 1974, L'univers politique des enfants, Paris, Presses de la Fondation nationale des sciences politiques.

- Pinquart M. et Silbereisen R. K., 2004, Transmission of values from adolescents to their parents: The role of value content and authoritative parenting, Adolescence, vol. 39, n 153:83-100.

- Searing D. D., Schwartz J. J. et Lind A.E., 1973, The structuring principle: political socialization and beliefs systems, American Political Science Review, vol. 67, n 1:415-432.

- Sears D. O. et Valentino N. A., 1997, Politics matters: Political events as catalysts for preadult socialization, Americain Political Science Review, vol. 91, n²1:45-65.

- Sigel R. S., 1989, Political learning in adulthood. A source book of theory and research, The University of Chicago Press, Chicago and London.

- Stoker L. et Jennings M. K., 1995, Life-cycle transitions and political participation: the case of marriage, American Political Science Review, vol. 89, $\mathrm{n}^{\circ}$ 2:421-433.

- Tedin K. L., 1974, The influence of parents on the political attitudes of adolescents, American Political Science Review, vol. 68, n 4:1579-1592.

- Tournier V., 2004, Générations politiques, in Le nouveau désordre électoral (sous la dir. de Cautrès B. et Mayer N.), Paris, Presses de Sciences Po:229-252.

- Tournier V., 2000, Filiation et politiques : la construction de l'identité et ses conséquences, in Les cultures politiques des Français (sous la dir. de Bréchon P., Laurent A. et Perrineau P.), Paris, Presses de Sciences Po:189-208.

- Tournier V., 1998, « La politique en héritage ? Socialisation, famille et politique : bilan critique et analyse empirique ", thèse de doctorat, Grenoble.

- Vaillancourt P. M., 1973, Stability of children's survey responses, Public Opinion Quarterly, vol. 37, $n^{\circ} 3: 373-387$

- Vollerbergh W. A. M., ledema J. et Raaijmakers Q. A., 2001, Intergenerational transmission and the formation of cultural orientations in adolescence and young adulthood, Journal of Marriage and the Family, vol. 63, n 4:1185-1198.

- Westholm A. et Niemi R. G., 1992, Political institutions and political socialization: A cross-national study, Comparative Politics, vol. 25, n 1:25-41. 